\title{
EVALUACIÓN DE COMPETENCIAS
}

Albert Arbós Bertran

\section{RESUMEN}

Al hablar de competencias en el mundo de la educación, nos encontramos ante un nuevo paradigma educativo, necesario para la calidad y para el incremento de la empleabilidad y la ciudadanía. ¿Qué elementos caracterizan este nuevo paradigma?

A una sociedad del conocimiento le corresponde una sociedad del aprendizaje, donde el centro del proceso educativo se mueve de la enseñanza al aprendizaje. El modelo educativo se centra, por tanto, en el estudiante y su capacidad de aprender; demanda más protagonismo para llegar a la información primaria, y al acceso y evaluación de esta información en sus formas más variadas (biblioteca, profesor, Internet, etcétera). Todo ello implica el cambio de rol del profesor/a, que asume el papel de supervisor/a y director/a del trabajo del estudiante y asesor/a del alumno que, a su vez, estructura el conocimiento y lo articula en conceptos claves.

Las competencias afectan a la definición de los objetivos, que guiarán el programa educativo a través de indicadores que nos darán la posibilidad de medirlos; ello implica unos objetivos dinámicos en función de las necesidades de la sociedad. Los resultados se reflejan en la evaluación del estudiante, que desplaza su objeto del simple conocimiento a las competencias y capacidades en función del proyecto educativo, y a través de estrategias de evaluación nuevas - portafolio, 
trabajo proyectos...- y viejas -pruebas escritas, orales...-. Todo ello comporta diversos ritmos individuales de trabajo que afectan a la organización del aprendizaje.

\section{APROXIMACIÓN AL CONCEPTO DE COMPETENCIA}

A partir de la década de los 70, se detecta un divorcio entre lo que enseña la escuela y lo que le pide la sociedad. Con la intención de superar esta divergencia, las competencias relacionan el mundo de la enseñanza/educación y las necesidades del mercado del trabajo, y, por extensión, las necesidades de la sociedad.

Por tanto, las primeras competencias en estudiarse fueron las laborales, que ligaban el "perfil profesional o cualificación" con las competencias. De ahí pueden surgir las primeras definiciones. Cualificación es la capacidad potencial para realizar unas tareas correspondientes a una actividad. Competencia es la capacidad real para lograr el resultado deseado en un contexto dado y con capacidad de transferencia. La competencia se manifiesta mediante comportamientos habituales y observables que permiten alcanzar el éxito en la tarea que se realice.

El interés primario de las competencias es que sean transferibles, esto implica responder a la pregunta: ¿para qué sirve lo que aprendemos? Este enfoque de las competencias se basa en la necesidad de evaluar más que el contenido de las materias: más allá del saber, conviene que los alumnos sepan hacer, estar y ser. Así, aparte de las competencias académicas, se incorporan las competencias del entorno laboral y del contorno social. Se entienden como conocer y comprender (conocimiento teórico), saber cómo actuar (aplicación a situaciones concretas) y saber cómo ser (valores que se integran en el contexto social). De este preámbulo, obtenemos una nueva definición de competencia (Martens, 1997): "Con el término competencia, entiendo el conjunto de saberes técnicos, metodológicos, sociales y participativos que se actualizan en una situación particular y en un momento particular». 


\section{ENFOQUES DE LAS COMPETENCIAS}

Podemos analizar, entre otros, tres grandes enfoques que marcan el análisis teórico en este campo y que nos revelan indicadores para su evaluación.

Análisis funcional. Mediante este análisis se describe el puesto o función, como conjunto de elementos de competencia, con criterios de evaluación con base en unos niveles minimos requeridos. "Las competencias son conocimientos, destrezas y aptitudes necesarias para ejercer una profesión y resolver los problemas profesionales de manera autónoma y flexible, con capacidad de colaborar en un entorno profesional y en la organización del trabajo" (Bunk, 1994).

"Las competencias representan una combinación dinámica de atributos, con relación a conocimientos, habilidades, actitudes y responsabilidades, que describen los resultados del aprendizaje de un programa educativo o lo que los alumnos son capaces de demostrar al final de un proceso educativo" (Proyecto Tuning) ${ }^{1}$.

Análisis conductista. Nos permite construir las competencias a partir del comportamiento de los individuos con mayor nivel de desempeño, partiendo de las personas que hacen un trabajo excelente de acuerdo a los resultados que espera la organización. "Las competencias son las características de fondo de un individuo que guardan una relación causal con el desempeño eficaz o superior del puesto de trabajo" (Boyatzis ${ }^{2}, 1995$ ).

Análisis constructivista. Basado en la Psicología cognitivista, nos plantea unas competencias que se elaboran desde las relaciones existentes entre los grupos y su entorno, además de entre las situaciones de trabajo y la capacitación requerida. “Competencia es la integración entre saber, saber

\footnotetext{
${ }^{1}[$ http://europa.eu.int/comm/education/tunong.html] y [http://www.let.rug.nl/TuningProject].

${ }^{2}$ BOYATZIS, R.E.; COWEN, S. y KOLB, D.A., Innovation and professional education.
} 
hacer y el saber ser" (Ibarra, 2000). Este concepto de competencia, el más moderno de los tres, implica factores de superación individual y grupal que permiten el desarrollo de los recursos personales para integrarlos en el entorno, en una interacción de enriquecimiento mutuo.

\section{¿QUÉ COMPETENCIAS?}

Después del breve marco teórico, deberíamos preguntarnos, ¿qué competencias debemos elegir, trabajar... evaluar? No es el objeto de este escrito, por ello expondremos un breve ejemplo, ¿qué competencias deberíamos considerar para los alumnos universitarios?

Dentro del mundo anglosajón, el modelo británico apunta a dos áreas: conocimientos y comprensión, por un lado, y competencias transversales, por otro. Para cada una de las áreas describe tres niveles de logro: Threshold, Good, Excellent. El modelo australiano, también señala tres dimensiones: Generic skills, attributes and values; Adquisition of a body of knowledge; y Professional/technical or other job related skills.

Por su parte, Clanchy y Ballard (1995) establecen cuáles deben ser las habilidades y actitudes que la educación universitaria ha de desarrollar en sus graduados: pensar y razonar; investigar (indagar, buscar y gestionar información) y comunicar (oral y escrito).

Para acreditar los programas de Ingeniería, el ABET Criteria pide a los graduados que posean:

- Habilidades para trabajar en equipos multidisciplinares.

- Habilidad para comunicarse efectivamente.

- Reconocimiento y habilidad para implicarse en el aprendizaje a lo largo de la vida.

- Comprensión de la responsabilidad profesional y ética.

- Habilidad para llevar a cabo experimentos, y analizar e interpretar datos. 
- Habilidad para identificar, formular y resolver problemas de Ingeniería... etcétera.

Existen otros muchos sistemas, como los de Birenbraum -competencias cognitivas, metacognitivas, sociales y afectivas-, Bennet -gestión de uno mismo, gestión de los otros, gestión de las tareas, gestión de la información-, y tantos otros, que deberían ser objeto de otro estudio. Pero con lo señalado nos hacemos una idea de la variedad de competencias y clasificaciones que las agrupan. Por tanto, cuando hagamos referencia a evaluación de las competencias, lo haremos de forma genérica y transferible de unas clasificaciones a otras. No obstante, cabe apuntar que cualquier plan de formación en las competencias, debería contener estos cuatro elementos:

1. Perfil de formación -competencias específicas y transversales o genéricas-. Debería romper con la imagen del aprendizaje como suma de adquisiciones sucesivas de contenidos para afirmar a un alumno -graduado- como una persona que cumple un perfil definido.

2. Objetivos del establecimiento educativo con relación a estas competencias.

3. Las materias, sus objetivos y su peso en el currículum -conocimientos-.

4. Criterios de certificación y evaluación de la calidad de la progresión académica.

\section{EVALUACIÓN DE COMPETENCIAS}

La evaluación es la manera de que todo lo que ocurre en el ámbito curricular no se quede en meros deseos o propuestas genéricas. Los docentes no innovarán su docencia si el proceso de enseñanza-aprendizaje no implica una modificación en las prácticas evaluadoras. Todos los objetivos que se pretenden en la innovación tienen que valorarse a través de la evaluación, de lo contrario, no se conseguirán. 
Respecto a los alumnos, nos permitirá ver de dónde partimos, cuáles son sus carencias y fortalezas en el ámbito individual y grupal. Los alumnos no modifican su forma de aprender si se les sigue evaluando según formas pretéritas.

En el ámbito de las competencias, nos obligará a operativizarlas -operativizar su desarrollo-, es decir, asignarles comportamientos concretos y observables. También nos facilitará saber si se están produciendo mejoras. Y nos dará pistas para rediseñar o redefinir las competencias clave, así como para mejorar los procedimientos de trabajo.

Podemos considerar, como premisa, la frase: "Todo lo que no se evalúa, se devalúa".

Mostramos, a continuación, un esquema que puede ayudarnos a visualizar el posible proceso de desarrollo y evaluación de las competencias:

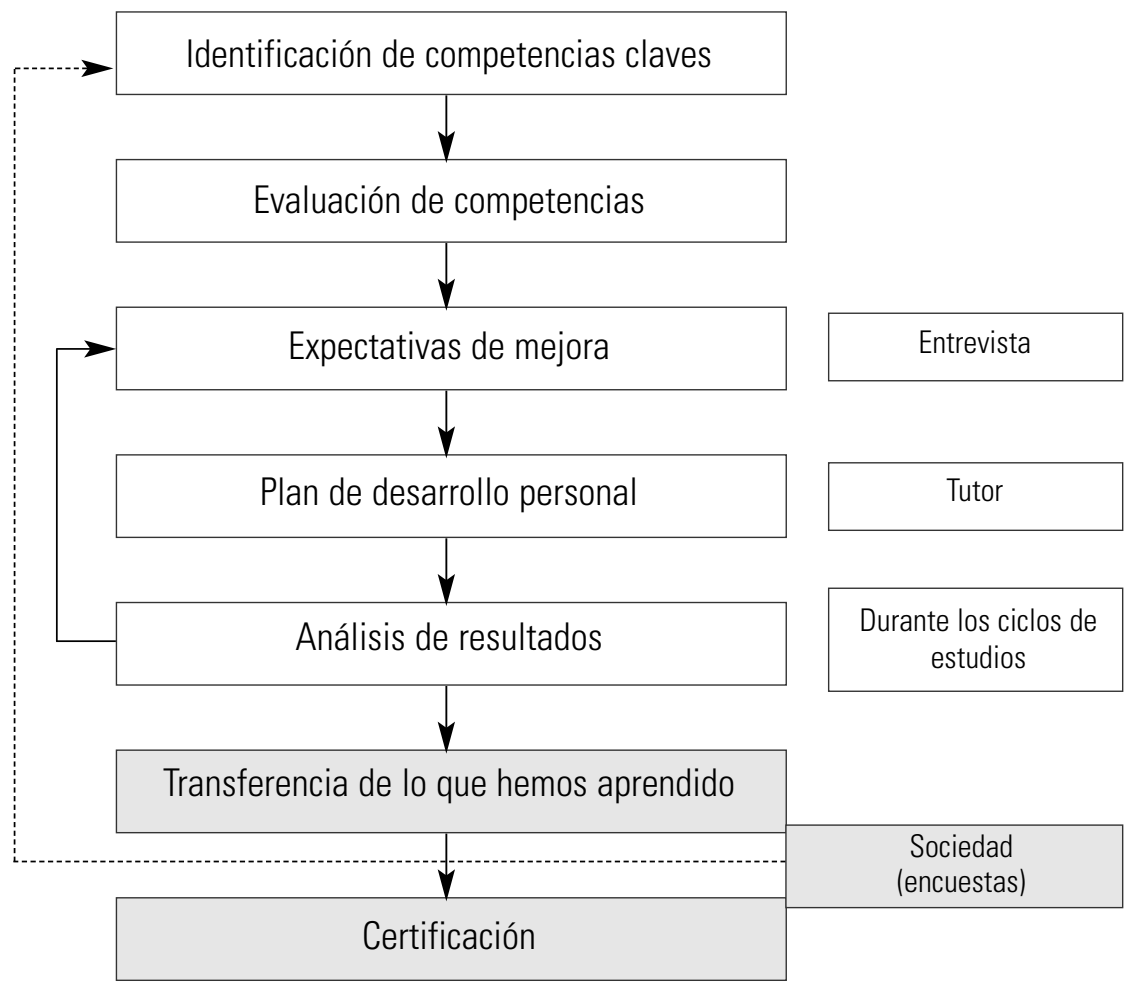


A raíz del esquema, podemos entrever algunas claves para la puesta en práctica de las competencias:

- Partimos de la idea central de que las competencias no son meros conocimientos, sino más bien habilidades y capacidades de carácter personal, compatibles y complementarias a los conocimientos teóricos y profesionales/laborales que se transmiten en todos los estadios del sistema educativo.

- Por ello, no pueden desarrollarse de la misma manera que los conocimientos. Al ser capacidades, no resulta adecuada la transmisión de profesor a alumno, sino que se requiere el esfuerzo personal del alumno para adquirirlas -aprendizaje activo- bajo la guía y ayuda de un tutor.

- Además, al incidir directamente en las características personales de los alumnos, variarán de unos a otros. Ello aconseja su desarrollo personalizado. Este desarrollo sugiere actuar en dos líneas complementarias: la introducción de metodologías docentes innovadoras y el fomento de las tutorías individuales.

- Algunas competencias pueden desarrollarse a través de la docencia de las asignaturas: trabajo en equipo, gestión del tiempo, comunicación, capacidad de análisis y de síntesis, etcétera. Pero otras competencias necesitan ser desarrolladas, al menos en parte, en sesiones de tutoría: plan personal de trabajo, autoconocimiento, compromiso ético, iniciativa, empatía, etcétera.

\section{¿QUÉ EVALUACIÓN?}

Entre los criterios de calidad de la evaluación, podemos citar con Zabalza ${ }^{3}$ (2001) y Yániz ${ }^{4}$ (2002):

${ }^{3}$ ZABALZA, M. A., "Evaluación de los aprendizajes en la Universidad"., en A. GARCÍA VALCARCEL., Didáctica Universitaria.

4 YÁNIZ, C., Evaluación del aprendizaje: concepto y planteamientos

básicos., Unidad didáctica para la formación del profesorado universitario, en [www.ice.deusto.es/alud/guia.asp]. 
- Debe servir para ayudar a los alumnos a desarrollar sus capacidades, por lo tanto, debe ser más formativa que acreditativa.

- Debe referirse a todos los objetivos formativos, es decir, ser integradora.

- Debe estar inmersa en el desarrollo habitual y del proceso de enseñanza-aprendizaje y coherente con el estilo de trabajo habitual.

- Debe ser una parte sustantiva del proceso formativo.

- Debe ser coherente con el estilo de trabajo en el aula.

- Debe ser inicial, procesual y final.

- Debe incorporar nuevas metodologías e instrumentos de evaluación.

- Debe incluir demandas cognitivas variadas y progresivas.

- Debe contener información previa -criterios- y posterior -orientación de los resultados-.

- Debe atender a procesos intelectuales más complejos que la memorización.

- Debe ser objetiva, entendida como posibilidad de justificación razonada.

- Debe estar vinculada a la calidad: de sí misma, de la institución...

- Puede incluir nuevas tecnologías como recurso para la evaluación.

- Pueden valorarse los aprendizajes adquiridos fuera del aula.

Debemos tender, en una palabra, a la evaluación auténtica (Wiggings, 1990) 5 . Vamos a comparar, brevemente, la evaluación auténtica con la mal llamada tradicional, ya que la evaluación clásica posee metodologías e instrumentos de gran utilidad, si son bien aplicados:

WIGgings, G., "The case for authentic assessment", en Practical Assessment, Research and Evaluation. 


\begin{tabular}{l|l} 
Evaluación auténtica & Evaluación tradicional \\
\hline Examen directo tareas complejas. & Ítems indirectos. \\
\hline Requiere desempeño. & Evocación fuera de contexto. \\
\hline Conjunto complejo de tareas. & Preguntas de única respuesta. \\
\hline Respuestas justificables. & Respuestas correctas. \\
\hline Validez y fiabilidad a través de criterios. & Una respuesta correcta. \\
\hline Validez implica capacidad en el mundo real. & Emparejar ítems con contenido curricular. \\
\hline Situaciones complejas. & Ejercicios mecánicos. \\
\hline Valoración de lo divergente. & Valoración de la convergencia preestablecida. \\
\hline
\end{tabular}

Para aplicar correctamente esta evaluación auténtica deberíamos observar, entre otros, los siguientes principios:

- Basarse en una concepción didáctica/educativa de la evaluación, es decir, debe ser inherente al proceso de enseñanza-aprendizaje; lo que trae como consecuencia plantear, en el ámbito organizativo, esta evaluación al inicio de la aplicación del currículum y no solamente al final.

- Continuidad y permanencia de la evaluación. Continuidad en las diversas fases -inicial, procesual y final- y permanencia en la toma de decisiones consecuentes con los distintos momentos evaluativos.

- Esta continuidad se garantiza con el feed back durante todo el proceso educativo, en el ámbito de profesor y de tutor.

- A pesar de que debemos combinar los diferentes roles de la evaluación -diagnóstica, formativa y sumativa-, deberíamos incentivar los roles diagnóstico y, sobre todo, formativo.

- La evaluación no es el punto final sino el inicio del proceso educativo. La evaluación no es una finalidad, es un medio para mejorar el aprendizaje de todos los actores del proceso educativo. 
- La evaluación no puede ser unidireccional -profesor-alumno-, debe ser el propio alumno quien asuma la responsabilidad global de aprender.

- La evaluación deberá relacionar lo aprendido -ahí entra de lleno la evaluación de las competencias- con la vida real.

- Todo ello nos lleva a una evaluación individual, aunque la administración de pruebas pueda ser grupal. En último término, y como objetivo-tendencia, deberíamos considerar una evaluación personalizada.

- Esta evaluación debe llevar a la reflexión de los profesores y de las instituciones educativas, para relacionar de forma eficaz la teoría y la práctica, en un proceso en espiral de investigación-acción.

- En el ámbito formal, las instrucciones deberán ser claras y los criterios de valoración apriorísticos, pero no inamovibles.

$\mathrm{Al}$ analizar la evaluación auténtica, podemos distinguir claramente cuatro elementos esenciales (Herrington y Herrington, 1998) ${ }^{6}$, que ya podemos aplicar a la evaluación de las competencias, a saber:

1. El alumno, que deberá actuar de forma eficaz -conseguir los objetivos propuestos- sobre los conocimientos adquiridos y producir resultados (debemos basarnos en el cambio del paradigma "consumo de saber" por el paradigma "producción de saber").

2. El contexto, que deberá reflejar las condiciones bajo las que tendrá lugar el desempeño.

3. La actividad auténtica, que implica desafíos complejos, situaciones poco estructuradas -¿debemos dar sólo los datos

${ }^{6}$ HERRINGTON, J. y HERRINGTON, A., "Evaluación auténtica y multimedia, ¿de qué manera los estudiantes responden a un modelo de evaluación auténtica?", en Higher Education Research and Development. 
de un problema o los datos que aparecen en la vida real-, que requieren un juicio valorativo -último escalón del pensamiento complejo, que ya nos propuso Bloom en los años 60 en su Taxonomía- y un conjunto de tareas complejas, engarzadas unas con otras que hagan al alumno protagonista del aprendizaje. Por supuesto la evaluación deberá estar integrada, como un todo sin solución de continuidad, en estas actividades.

4. Los indicadores ${ }^{7}$, indispensables para una valoración objetiva, o mejor, intersubjetiva, procurarán la validez y la fiabilidad de los instrumentos, con criterios adecuados para calificar/valorar la variedad de producciones del alumnado, fruto de una variedad de procedimientos evaluativos que nos ayudarán a recoger la información necesaria.

\section{¿QUÉ, CÓMO Y QUIÉN EVALÚA LAS COMPETENCIAS?}

Con lo expuesto en el párrafo anterior, algunas de las preguntas ya podrían quedar contestadas, al menos en sus bases generales. Puntualicemos algunos detalles.

Partimos de la premisa de que debe evaluarse el contenido-seminarios intensivos o extensivos- de la competencia y

${ }^{7}$ Ejemplo de indicadores desglosado en ítems para la evaluación de la competencia:

\section{Responsabilidad en el trabajo:}

Nivel de alarma:

Nivel presente:

Bastante presente:

Excelente:
No se responsabiliza de sus tareas ni obligaciones.

No acepta las consecuencias de sus decisiones.

Por iniciativa propia toma muy pocas decisiones.

No tiene cuidado de sus materiales.

No mantiene los compromisos adquiridos.

Prefiere trabajar bajo control.

Mantiene los acuerdos.

Planifica su trabajo.

Delante de un problema compara las diferentes soluciones posibles.

Interpreta los mensajes y, según su importancia, da respuestas adecuadas.

Asume las consecuencias de sus actos.

Distribuye muy bien su tiempo.

Participa teniendo siempre presente los objetivos de la organización.

Cuando es necesario, se autocontrola y se adapta. 
su aplicación -integración de las competencias en las asignaturas o materias- a fin de que entren/entremos en un proceso de mejora continua. Cada competencia podría tener un sistema de evaluación: autoevaluación ${ }^{8}$, coevaluación ${ }^{9}$, heterevaluación ${ }^{10}$ o evaluación externa. ¿De que dependería?: de los objetivos propuestos, de la metodología de aprendizaje y del plan de innovación pedagógica para comprobar el nivel adquirido en la competencia.

\section{PAPEL DEL TUTOR}

\begin{tabular}{|l|l|l|l|}
\hline $\begin{array}{l}\text { Tutor de competencia } \\
\text { como contenido }\end{array}$ & & Evaluación externa \\
\hline Tutor académico & Coevaluación & Heteroevaluación & \\
\hline Tutor empresa & Coevaluación & Heteroevaluación & \\
\hline
\end{tabular}

De los sistemas de evaluación, creemos que el más interesante en la evaluación de las competencias es la autoevaluación, ya que coloca el énfasis en el papel activo del alumno en su aprendizaje y fomenta la reflexión sobre el mismo.

\section{¿CON QUÉ INSTRUMENTOS EVALUAR LAS COMPETENCIAS?}

Partimos de la base que ningún instrumento es bueno o malo en sí, sino que depende del uso -finalidad- que le demos y de la correcta aplicación -aspectos formales que garantizan su calidad- que hagamos. Empecemos por el más clásico: las pruebas escritas.

8 La autoevaluación se produce cuando el sujeto evalúa sus propias actuaciones.

9 La coevaluación consiste en la evaluación mutua, conjunta, de una actividad o un trabajo determinado realizado entre varios.

${ }^{10}$ La heteroevaluación consiste en la evaluación que realiza una persona sobre otra: su trabajo, su actuación, su rendimiento, etcétera. 


\section{Pruebas escritas. Criterios de calidad:}

a) Vincular la evaluación al aprendizaje mediante las pruebas escritas.

b) Fomentar la implicación de los alumnos en el proceso de evaluación.

c) Evaluación multidimensional.

d) Utilización de criterios de evaluación, específicos ${ }^{11} \mathrm{O}$ genéricos ${ }^{12}$, transparentes, es decir, predeterminados y conocidos por profesores y alumnos.

Pruebas objetivas. Criterios de calidad:

a) Su finalidad debe ser la de discriminar conceptos, terminología... que consideremos como más importantes y básicos para progresar en una materia. Puede ser útil, por tanto, en una evaluación inicial para diagnosticar lo que sabe un alumno del tema que vamos a estudiar.

b) Contemplar la posibilidad de que los propios alumnos generen preguntas, lo que provocará que trabajen los contenidos con una motivación añadida.

${ }^{11}$ Ejemplo de criterios específicos de valoración de respuestas a preguntas abiertas (Morales, 1995):

10.- Respuesta extraordinaria, hay originalidad, muestra pensamiento independiente, etcétera.

9.- Óptima organización, aporta en la respuesta toda la información que se puede esperar.

8.- Buena organización de lo más importante.

7.- Buen uso de lo más importante.

6.- Muestra comprensión, pero poca información.

5.- Hay elementos relevantes, pero no muestra una comprensión adecuada.

4.- No responde realmente a la pregunta, muestra cierta comprensión general del tema.

3.- Confuso, mal organizado, no hay nada relevante.

${ }^{12}$ Ejemplo de criterios genéricos de valoración de respuestas a preguntas abiertas (Morales, 1995) -se explicitan las puntuaciones máximas-:

Información básica (2); información adicional (2); ejemplos y anotaciones personales (2); demuestra comprensión (2); bien estructurado, claridad (1); ortografía y presentación (1). 
c) Criterios de evaluación claros y transparentes. En ellos podemos vehicular elementos adicionales que nos permitan conocer otras facetas del alumno ${ }^{13}$.

d) Análisis de ítems y distractores que facilitan una evolución constante para la mejora de la fiabilidad y validez de los resultados.

Realización de proyectos (cooperativos o Bachelor Thesis). Los proyectos pueden elaborarse a título individual o conjuntamente con otros alumnos o, incluso, el mismo profesor. A ser posible, apostamos por esta segunda modalidad. Criterios de calidad:

a) Definen conjuntamente el proyecto, los objetivos del mismo; comparten las diversas fases de la elaboración y son coautores del informe final. Por tanto, hay un compromiso real en todas las fases del proyecto.

b) Se producen dos aspectos de carácter simultáneo: la evaluación y la formación y desarrollo personal de todos y cada uno de los participantes.

Elaboración de matrices. Las matrices son tablas de doble entrada, filas y columnas que representan información que puede ser descriptiva y explicativa. Las filas y columnas pueden incluir los aspectos y características más variadas del objeto evaluado. Criterios de calidad:

a) Se deben plantear las matrices en función de los objetivos que pretendamos en la evaluación. En nuestro caso, aconsejamos la matriz ordenada en función de la persona, sobre todo las matrices descriptivas.

\footnotetext{
${ }^{13}$ Vamos a reseñar una posibilidad, a título de ejemplo:

Podríamos añadir, al lado de cada respuesta de opción múltiple, otra sobre el grado de seguridad sobre la misma:

1. Estás completamente seguro; 2. Estás bastante seguro; 3. Estás poco seguro; 4. Estás nada seguro.

En función del acierto o error en la respuesta y de haber optado por alguno de los cuatro grados de seguridad, podríamos establecer una baremación como la que sigue: Acierto $+1=$ puntuación $* 2 /$ Acierto $+2=$ puntuación $* 1.5 /$ Acierto $+3=$ puntuación * $1 /$ Acierto $+4=$ puntuación * 0.5

Error $+1=$ puntuación $-1 /$ Error $+2=$ puntuación $-0.5 /$ Error $+3=$ puntuación $/$ Error $+4=$ puntuación +0.5
} 
b) Las matrices explicativas son muy útiles para orientar el aprendizaje ya que nos proporcionan explicaciones, razones y causas del objeto evaluado.

c) Las matrices deben diseñarse de tal manera que permitan los análisis posteriores: visión global, análisis detallado, combinación de datos paralelos, etcétera.

Ejercicios de simulación. La simulación es un tipo específico de evaluación por el que se trata de representar la realidad de una forma simplificada. Los ejercicios de simulación cuentan con una serie de inputs o datos de partida y una serie de outputs o resultados que se desprenden una vez elaborados. Criterios de calidad:

a) Es preferible utilizar el método deductivo, es decir, deducir implicaciones de los ejercicios propuestos, realizar una serie de predicciones y contrastarlas con la realidad.

b) Debemos incitar a las respuestas ad boc de los alumnos y huir de las respuestas estandarizadas; por tanto, debemos evitar plantear problemas habituales que comportan respuestas estandarizadas.

Los métodos de simulación, aunque muestran importantes virtudes, no han tenido un desarrollo importante en el ámbito de las ciencias sociales, debido probablemente al escepticismo de una gran parte de los investigadores, pero debemos hacer un esfuerzo de superación del mismo ya que sus ventajas en el ámbito de la evaluación de competencias superan con creces los inconvenientes.

Escalas de observación. Las escalas son instrumentos utilizados para determinar diferencias de grado o intensidad respecto a algún objetivo, en este caso, competencial. Criterios de calidad:

a) Debe tenerse en cuenta el contexto de cada aplicación y el criterio con el que se ha construido. Este criterio permite establecer diferencias de grado entre individuos, respecto a la competencia observada.

b) El modo más adecuado para llevarla a cabo es que varias personas -por ejemplo, la triangulación- sitúen al individuo en la escala del objeto evaluado. 
c) Si se utiliza la autoevaluación, requiere que el individuo sea consciente de sus convicciones y sentimientos respecto al objeto evaluado y esté dispuesto a exponerlas con sinceridad. Debe conocer con detalle y objetividad el significado de los distintos grados de la escala.

Presentaciones orales. Criterios de calidad:

a) Valoración ponderada de sus dos grandes finalidades: dominio sobre el contenido y capacidad de comunicación oral.

b) Determinar, previamente, si el soporte será únicamente oral o se podrán utilizar medios tecnológicos -transparencias, presentaciones con medios informáticos y/o audiovisuales-. Los criterios de valoración deberán contender la valoración de los medios utilizados.

c) Los criterios de valoración deberán combinar la valoración de aspectos tangibles ${ }^{14}$-conocimientos, información, análisis e interpretación, juicio y valoración- y elementos

${ }^{14}$ Criterios para evaluar una presentación informativa, según Quigley (1998):

a) Introducción (15 puntos):

- El orador consiguió atraer la atención y el interés de la audiencia.

- El orador estableció la finalidad y la idea central del discurso.

- El orador realizó una presentación breve del resto del discurso.

b) Cuerpo (40 puntos):

Adaptación a la audiencia.

- El orador ajustó el mensaje a los intereses de la audiencia.

- El orador utilizó un lenguaje y ejemplos apropiados para la audiencia.

Razonamiento.

- Presentó los puntos principales de un modo claro y preciso.

- Presentó los puntos en un orden lógico y fácil de seguir.

- Desarrolló suficientemente los principales puntos.

- Recurrió, al menos, a dos fuentes externas de investigación.

Organización.

- La presentación tuvo una introducción, un cuerpo y una conclusión.

- Las transiciones dentro de la presentación fueron fáciles de seguir.

- El orador completó el guión escrito que había preparado.

c) Conclusión (15 puntos):

- El orador preparó a la audiencia para el final de la presentación.

- Al final resumió y reforzó los puntos principales.

- Al final reforzó la idea central de la presentación.

d) Presentación (30 puntos):

- Estaba planificada, pero resultaba coloquial.

- El orador mantuvo un contacto visual frecuente con la audiencia.

- El ritmo, la claridad y el volumen del discurso lo hicieron comprensible.

- Los gestos reforzaron el mensaje verbal.

- El discurso se desarrolló en el intervalo de tiempo previsto. 
intangibles -actitud, empatía y cualquier elemento del ámbito de la comunicación-.

Memorias analíticas, son simulaciones en las que el alumno redacta un problema concreto -un par de páginas-, analizando la situación para favorecer la toma de decisiones. Criterios de calidad:

a) Aunque obtenidas de los ámbitos relacionados con la gestión y la administración públicas, deben plantearse a alumnos de enseñanza postsecundaria, preferentemente universitarios. Encaja en estudios económico-administrativos o en materias cercanas a la organización, por ejemplo, organización escolar en la Ciencia de la Educación.

b) Deberían valorarse dos dimensiones: la capacidad de plantear el análisis de una manera clara, concisa y precisa, y las estrategias y técnicas concretas relativas a la asignatura en la que estemos inmersos.

Mapas conceptuales, a través de los que se muestran los elementos de razonamiento que utiliza el alumno y las relaciones que establece entre ellos. Son especialmente adecuados cuando existe una fuerte carga conceptual en el aprendizaje y para la evaluación diagnóstica de conceptos previos. Criterios de calidad:

a) Combinar criterios de corrección. Por un lado, los formativos de corrección, en los que se muestren los aspectos correctos, los que son mejorables y cómo pueden optimizarse estos últimos. De otro, los cuantitativos ${ }^{15}$, que nos darán una puntuación diferenciada.

${ }^{15}$ A título de ejemplo citamos algunos criterios apuntados por Belmonte (1997): Número de niveles; conectores; relaciones cruzadas; número de conectores distintos, número de conceptos diferentes correctamente incluidos; grado de ramificación. 
Diarios de doble entrada, en los que el alumnado escribe ideas, frases y argumentos relevantes de su tarea y el significado personal que esto tiene para él (Angelo y Collins, 1993) ${ }^{16}$; el alumno dialoga con el texto y explora sus reacciones a la lectura. Nos permite conocer los intereses, preocupaciones y valores de los alumnos. Criterios de calidad:

a) Es adecuado cuando es importante el estudio detallado de textos, preferentemente cortos, pero relevantes para la asignatura.

Portfolio: el alumno acumula evidencias diversas del proceso y del resultado de su aprendizaje (Mullin, 1998) ${ }^{17}$. Debe explicar el aprendizaje que ha supuesto cada evidencia; las evidencias pueden ser de diversa naturaleza. Criterios de calidad:

a) Estructura previamente pactada entre alumno y profesor. Esta estructura puede ser de diverso grado y puede establecer lo obligatorio y lo optativo.

b) El alumno debe seleccionar las evidencias que quiere aportar, que vendrán marcadas por los objetivos de la asignatura.

c) Elaborar los criterios de evaluación -cómo será evaluado, presentación y defensa final, entregas parciales, reflexiones sobre el proceso y el producto o resultadoconjuntamente entre profesor y alumno.

d) Debería permitir desarrollar destrezas de colaboración entre los alumnos para mejorar, entre otros, la autoestima y la generosidad, la motivación y la ayuda mutua; en caso contrario, puede fomentar actitudes egoístas.

${ }^{16}$ ANGELO, T.A. y COLLINS, K.P., Classroom assessment techniques.

${ }^{17}$ MULlin, J.A., "Portfolios: purposeful collections of student work", en ANDERSON, R.S., y SPECK, B.W. (Eds.)., Changing the way we grade student performance: Classroom assessment and the new learning paradigm. 
Discusión en grupo, puede ser en forma presencial o a través de Internet, en un forum de discusión, donde, por ejemplo, un grupo lo presenta, otro lo critica y otro lo intenta mejorar. Criterios de calidad:

a) La subjetividad de este tipo de evaluación se debe contrarrestar con la utilización de registros sistemáticos y plantillas de evaluación. Este criterio de objetividad puede suavizarse con la participación de los alumnos en su elaboración. También puede combinarse con la autoevaluación.

b) Al valorar la participación, deberemos fundamentar nuestras/sus valoraciones; distinguir el grado de aportación de contenidos y propuestas, así como la penalización por "infracciones éticas".

c) Juega un papel muy importante el animador u observador no participante, que de vez en cuando va informando al grupo de sus observaciones. En ningún caso debe intentar dirigir al grupo sino orientar la dinámica del mismo.

¿Cuáles son las principales reglas de juego? No he pretendido, con este escrito, que el lector, en su trabajo de evaluador, sea ya más eficaz, más prudente, más lúcido. Espero, en todo caso, haber realizado un trabajo de apertura... Apertura sobre nuevas perspectivas, permitiendo ver la evaluación no como una simple medida, como un simple juicio, como otro discurso. Apertura a las prácticas más simples a la vez que eficaces, más innovadoras y dinámicas. 


\section{REFERENCIAS BIBLIOGRÁFICAS}

ABET., Criteria for accrediting engineering programs., [www.abet.org] ., 2000.

BELMONTE, M., Mapas conceptuales y uves heurísticas de Gowin. Técnicas para todas las áreas de la enseñanza media., 1997. ANGELO, T.A. y COLLINS, K.P., Classroom assessment techniques., San Francisco., Jossey-Bass Publishers., 1993. BENCHMARK STATEMENT FOR HISTORY., Quality Assurance Agency for Higher Education (QAA). UK: ISBN: 1-85824-485-4., 2000 .

BENNET, N.; DUNNE, E. y CARRÉ, C., "Patterns of core generic skill provision in higher education"., Higher Education, No. 37. pp.71-93., 1999.

BOYATZIS., R.E.; COWEN, S. y KOLB, D.A., Innovation and professional education., San Francisco., Jossey Bass., 1995.

HERRINGTON, J. y HERRINGTON, A., “Evaluación auténtica y multimedia, ¿de qué manera los estudiantes responden a un modelo de evaluación auténtica?"., Higher Education Research and Development, No. 17., 3., pp.305-322., 1998.

MORALES, P., Tipos de pruebas: los exámenes orales y las preguntas de respuesta abierta., Bilbao., Universidad de Deusto., 1995.

MULLIN, J.A., "Portfolios: purposeful collections of student work", Changing the way we grade student performance: Classroom assessment and the new learning paradigm., San Francisco., R.S. Anderson y B.W. Speck (Eds)., Jossey Bass Publishers., 1998.

QUIGLEY, B.L., en Changing the way we grade student performance: Classroom assessment and the new learning paradigm., San Francisco., R.S. Anderson y B.W. Speck (Eds)., Jossey Bass Publishers., 1998. 
WIGGINGS, G., "The case for authentic assessment"., Practical Assessment, Research and Evaluation, No. 2 (2). Disponible en Internet: http//ericae.net/getvn.asp?v=2\&n=2., 1990.

YÁNIZ, C., Evaluación del aprendizaje: concepto y planteamientos básicos., Unidad didáctica para la formación del profesorado universitario.,www.ice.deusto.es/alud/guia.asp., 2002.

ZABALZA, M. A., "Evaluación de los aprendizajes en la Universidad», en GARCÍA VALCARCEL, A., Didáctica Universitaria., Madrid., La Muralla., 2001.

[http///europa.eu.int/comm/education/tuning.html]. [http//www.let.rug.nl/TuningProject]. 